\title{
Helicity Modulus and Meissner Effect in a Fluctuating Type II Superconductor
}

\author{
Tao Chen and S. Teitel \\ Department of Physics and Astronomy, University of Rochester, Rochester, New York 14627
}

(June 24, 2021)

\begin{abstract}
The helicity modulus for a fluctuating type II superconductor is computed within the elastic medium approximation, as a probe of superconducting phase coherence and the Meissner effect in the mixed state. We argue that at the vortex line lattice melting transition, there remains superconducting coherence parallel to the applied magnetic field, provided the vortex line liquid retains a finite shear modulus at finite wavevector.

74.60.Ge, 64.60-i, 74.40.+k
\end{abstract}

Typeset using REVTEX 
In the high temperature superconductors, fluctuation effects are believed to be important over a wide region of the $H-T$ phase diagram [1 [5]. Recently there has been much controversy concerning the effect of vortex line fluctuations on long range order of the superconducting wavefunction in the mixed state [4]. Part of this controversy has concerned the definition of the proper gauge invariant correlation function. In this work we reconsider the question of superconducting coherence by considering instead the behavior of the helicity modulus [9] of the superconductor, which has proven a valuable criteria for coherence in related superfluid [10] and Josephson array [11] models. As will be seen below, it is an intrinsically gauge invariant quantity. Furthermore, the helicity modulus is equivalent to the linear response coefficient between an applied perturbation in magnetic field and the resulting supercurrent. Hence it more directly probes one of the most characteristic properties of a superconductor, the partial Meissner effect of the mixed state.

Working in the elastic medium approximation, we compute the helicity modulus to lowest order in the fluctuation of vortex lines. We show that vortex line fluctuations have a dramatically different effect on the helicity modulus parallel versus transverse to the applied magnetic field. For the parallel case, the helicity modulus gives total screening as in the Meissner effect. We argue that this total screening is unaffected by the vortex line lattice melting transition, provided the vortex liquid retains a finite shear modulus at finite wavevector. In the limit of an extreme type II superconductor with $\lambda \rightarrow \infty$, our results explain recent numerical simulations by Li and Teitel [12] which show clearly the persistence of phase coherence parallel to the applied field, well into the vortex liquid state. Similar results were obtained by Feigel'man et al. [13], working with a related $2 d$ boson model. We show a simple relation between their results and ours, which can be expressed in terms of the "winding number" of vortex lines. For simplicity, we carry out our calculation for an isotropic superconductor; the extension to the uniaxial anisotropic case is straightforward. We work within the London approximation, which should be valid provided one is not too close to $H_{c 2}$.

The Landau-Ginzburg Helmholtz free energy [14] for an isotropic uniform superconduc- 
tor, within the London approximation of constant wavefunction amplitude, can be written as,

$$
\mathcal{H}=\frac{1}{2} J_{0} \int d^{3} r\left\{|\nabla \theta-\mathbf{A}|^{2}+\lambda^{2}|\nabla \times \mathbf{A}|^{2}\right\}
$$

where $\theta$ is the phase of the superconducting wavefunction, $\lambda$ is the "bare" magnetic penetration length, $J_{0}=\phi_{0}^{2} / 16 \pi^{3} \lambda^{2}$ with $\phi_{0}$ the flux quantum, and $\left(\phi_{0} / 2 \pi\right) \mathbf{A}$ is the magnetic vector potential. $\nabla \times \mathbf{A}=2 \pi \mathbf{f}$ where $\mathbf{f}(\mathbf{r}) \equiv \mathbf{B}(\mathbf{r}) / \phi_{0}$ is the local density of magnetic flux quanta. The partition function $Z$ is computed averaging over independently 15 fluctuating $\theta$ and A, subject to the constraint that $\langle\mathbf{f}(\mathbf{r})\rangle=f \hat{\mathbf{z}}$ for a uniform average magnetic induction $B \hat{\mathbf{z}}$. In evaluating Eq.(1), the integration is to be cut off at the core of a vortex line, so that the energy stays finite.

In terms of the supervelocity $\mathbf{v} \equiv \nabla \theta-\mathbf{A}$, and its Fourier transform $\mathbf{v}_{q} \equiv \int d^{3} r \mathrm{e}^{i \mathbf{q} \cdot \mathbf{r}} \mathbf{v}(\mathbf{r})$, the helicity modulus is defined [10] as the linear response coefficient between induced supercurrent $J_{0} \mathbf{v}$ and an applied twist in phase. If we take $\mathbf{v}_{q} \rightarrow \mathbf{v}_{q}+\delta v_{q} \hat{\mu}$ in Eq.(1), then the helicity modulus in direction $\hat{\mu}$ is,

$$
\left.\Upsilon_{\mu}(\mathbf{q}) \equiv \frac{\partial^{2} \mathcal{F}}{\partial\left(\delta v_{q}\right)^{2}}\right|_{\delta v_{q}=0}=J_{0}\left\{1-\frac{J_{0}}{V T}\left\langle v_{q \mu} v_{-q \mu}\right\rangle\right\}
$$

where $\mathcal{F}=-T \ln Z$ is the total free energy, and $V=L_{z} L_{\perp}^{2}$ is the system volume. Because of the symmetry with which $\nabla \theta$ and $\mathbf{A}$ enter $\mathbf{v}, \Upsilon_{\mu}$ equivalently gives the induced supercurrent that flows in response to an applied perturbation in magnetic field, given by the vector potential $\delta \mathbf{A}_{q}=\delta v_{q} \hat{\mu}$. In evaluating $\Upsilon_{\mu}(\mathbf{q})$, the physically relevant case is the limit $q_{\mu} \rightarrow 0$. This follows from the convention of Baym [16], where to describe a system with a current flowing in the direction $\hat{\mu}$, the appropriate thermodynamic limit is to take the system size $L_{\mu} \rightarrow \infty$ first, followed by $L_{\nu} \rightarrow \infty$ for the directions $\hat{\nu} \perp \hat{\mu}$ (equivalently, $\mathbf{q} \cdot \delta \mathbf{A}_{q}=0$ in the London gauge).

Defining the vortex line density $\mathbf{n}$ by $\nabla \times \nabla \theta=2 \pi \mathbf{n}$, one can write an arbitrary configuration $\mathbf{v}_{q}$ in gauge invariant form as,

$$
\mathbf{v}_{q}=2 \pi i\left\{\mathbf{q} \chi_{q}+\frac{\mathbf{q} \times\left(\mathbf{n}_{q}-\mathbf{f}_{q}\right)}{q^{2}}\right\} .
$$


$\chi$ is a smooth function which gives the longitudinal part of $\mathbf{v}_{q}$, while the transverse part of $\mathbf{v}_{q}$ is determined by $\nabla \times \mathbf{v}=2 \pi(\mathbf{n}-\mathbf{f})$. Substituting Eq.(3) into the Hamiltonian (11), and decoupling the $\mathbf{n}_{q}$ and $\mathbf{f}_{q}$ degrees of freedom by completing the square in $\mathbf{f}_{q}$, we get

$$
\mathcal{H}=\frac{2 \pi^{2} J_{0}}{V T} \sum_{q}\left\{q^{2} \chi_{q} \chi_{-q}+\frac{1}{q^{2}}\left(1+\lambda^{2} q^{2}\right) \delta \mathbf{f}_{q} \cdot \delta \mathbf{f}_{-q}+\frac{\lambda^{2}}{1+\lambda^{2} q^{2}} \mathbf{n}_{q} \cdot \mathbf{n}_{-q}\right\}
$$

where $\delta \mathbf{f}_{q} \equiv \mathbf{f}_{q}-\mathbf{f}_{q}^{0}$ is the fluctuation of the magnetic flux density away from the value $\mathbf{f}_{q}^{0}=\mathbf{n}_{q} /\left(1+\lambda^{2} q^{2}\right)$, which minimizes the Hamiltonian for a given vortex configuration $\mathbf{n}_{q}$. The partition sum is now an average over all smooth functions $\chi$, all $\delta \mathbf{f}_{q}$ such that $\mathbf{q} \cdot \delta \mathbf{f}_{q}=0$ ( so that $\nabla \cdot \mathbf{B}=0$ ), and all vortex configurations $\mathbf{n}$. The vortex line interaction in Eq.(4) is just the familiar London result [14,17].

The helicity modulus is evaluated by substituting Eq.(3) into Eq.(2), and using the Hamiltonian (田) to evaluate the averages over $\chi_{q}$ and $\delta \mathbf{f}_{q}$. Taking the limit $q_{\mu} \rightarrow 0$ one gets,

$$
\lim _{q_{\mu} \rightarrow 0} \Upsilon_{\mu}(\mathbf{q})=\lim _{q_{\mu} \rightarrow 0} \frac{J_{0} \lambda^{2} q^{2}}{1+\lambda^{2} q^{2}}\left\{1-\frac{4 \pi^{2} J_{0} \lambda^{2}}{V T} \frac{(\hat{\mu} \times \hat{\mathbf{q}})_{\alpha}(\hat{\mu} \times \hat{\mathbf{q}})_{\beta}\left\langle n_{q \alpha} n_{-q \beta}\right\rangle}{1+\lambda^{2} q^{2}}\right\} .
$$

For a superfluid or spin model [9 11] phase coherence is indicated by a non-vanishing $\Upsilon_{\mu}$ in the limit $\mathbf{q} \rightarrow 0$. For the superconductor however, the gauge field $\mathbf{A}$ is free to adjust itself to screen out the applied phase twist (or perturbation $\delta \mathbf{A}$ ), and so even in the superconducting state $\Upsilon_{\mu}(\mathbf{q} \rightarrow 0) \sim q^{2}$, as seen in Eq.(5) above [18. In fact, if no vortex lines are present $\left(\mathbf{n}_{q}=0\right)$, Eq.(5) just gives the familiar total screening response of the Meissner state [16]. With the presence of vortex lines in the mixed state, we can generalize the form of the Meissner response, by defining a renormalized coupling $\left(J \lambda^{2}\right)_{R}$ and penetration length $\lambda_{R}$ such that

$$
\lim _{q \rightarrow 0} \Upsilon_{\mu} \equiv \frac{\left(J \lambda^{2}\right)_{R} q^{2}}{1+\lambda_{R}^{2} q^{2}}
$$

where $\lambda_{R}$ and $(J \lambda)_{R}$ may depend on the direction $\hat{\mathbf{q}}$. Thus to examine superconductivity it is necessary to consider the form of $\Upsilon_{\mu}$ at small but finite $\mathbf{q}$.

At high $T$, one can make a hydrodynamic approximation [19] and average over $\mathbf{n}(\mathbf{r})$ as if it was a continuous function, subject to the constraint that vorticity is conserved $\mathbf{q} \cdot \mathbf{n}_{q}=0$. 
Using the Hamiltonian (4) one gets $\Upsilon_{\mu}(\mathbf{q})=0$ as expected. At low $T$, one can evaluate the vortex line correlations using the elastic approximation for small fluctuations about a vortex line lattice. If $\mathbf{u}_{i}(z)$ is the transverse displacement of vortex line $i$ at height $z$ from its position $\mathbf{R}_{i}$ in the vortex line lattice, then $\mathbf{n}\left(\mathbf{r}_{\perp}, z\right)=\sum_{i} \delta\left(\mathbf{r}_{\perp}-\mathbf{R}_{i}-\mathbf{u}_{i}(z)\right)\left(\hat{\mathbf{z}}+d \mathbf{u}_{i} / d z\right)$. To evaluate Eq.(5) to lowest order in $T$, it is only necessary to consider the expansion of $\mathbf{n}_{q}$ to linear order in $\mathbf{u}_{i}$. For small $q>0$ we have,

$$
\mathbf{n}_{q}=i f\left(\mathbf{q} \cdot \mathbf{u}_{q} \hat{\mathbf{z}}-q_{z} \mathbf{u}_{q}\right)
$$

where $\mathbf{r}_{\perp}, \mathbf{R}_{i}$, and $\mathbf{u}_{i}$ lie in the $x y$ plane, $q_{z}$ and $\mathbf{q}_{\perp}$ are the components of $\mathbf{q}$ parallel and perpendicular to $\hat{\mathbf{z}}$, and $\mathbf{u}_{q} \equiv f \sum_{i} \int d z \mathrm{e}^{i\left(q_{z} z+\mathbf{q}_{\perp} \cdot \mathbf{R}_{i}\right)} \mathbf{u}_{i}(z)$. Correlations of $\mathbf{u}_{q}$ may be evaluated using the elastic Hamiltonian, as derived by Brandt [17],

$$
\mathcal{H}_{e l}=\frac{1}{2 V} \sum_{q}\left\{\left(c_{44} q_{z}^{2}+c_{11} q_{\perp}^{2}\right) u_{q L} u_{-q L}+\left(c_{44} q_{z}^{2}+c_{66} q_{\perp}^{2}\right) u_{q T} u_{-q T}\right\}
$$

where $u_{q L}$ and $u_{q T}$ are the components of $\mathbf{u}_{q}$ parallel and transverse to $\mathbf{q}_{\perp}$, and $c_{44}(\mathbf{q}), c_{11}(\mathbf{q})$, and $c_{66}(\mathbf{q})$ are the tilt, compression, and shear moduli respectively.

Substituting Eq.(7) into Eq.(5), and evaluating the displacement correlations using $\mathcal{H}_{e l}$, we find for perpendicular and parallel responses,

$$
\begin{aligned}
& \lim _{q_{x} \rightarrow 0} \Upsilon_{x}(\mathbf{q})=\lim _{q_{x} \rightarrow 0} \frac{J_{0} \lambda^{2} q^{2}}{1+\lambda^{2} q^{2}}\left\{1-\frac{B^{2}}{4 \pi\left(1+\lambda^{2} q^{2}\right)} \frac{q^{2}}{\left(c_{44} q_{z}^{2}+c_{11} q_{\perp}^{2}\right)}\right\} \\
& \lim _{q_{z} \rightarrow 0} \Upsilon_{z}(\mathbf{q})=\lim _{q_{z} \rightarrow 0} \frac{J_{0} \lambda^{2} q^{2}}{1+\lambda^{2} q^{2}}\left\{1-\frac{B^{2}}{4 \pi\left(1+\lambda^{2} q^{2}\right)} \frac{q_{z}^{2}}{\left(c_{44} q_{z}^{2}+c_{66} q_{\perp}^{2}\right)}\right\} .
\end{aligned}
$$

For the transverse response $\Upsilon_{x}(\mathbf{q})$ there are two cases to consider: $(i) \mathbf{q}=q \hat{\mathbf{z}}$, and $(i i)$ $\mathbf{q}=q \hat{\mathbf{y}}$. In $(i)$ the perturbation $\delta \mathbf{A}_{q}$ gives a magnetic induction along $\hat{\mathbf{y}}$, oscillating in the $\hat{\mathbf{z}}$ direction. It is thus a tilt modulation of the original induction $B \hat{\mathbf{z}}$. In $(i i)$, the perturbation gives a magnetic induction along $\hat{\mathbf{z}}$, which oscillates along $\hat{\mathbf{y}}$; it is thus a compression modulation of $B \hat{\mathbf{z}}$. Accordingly, Eq.(9) shows that in $(i) \Upsilon_{x}$ depends on $c_{44}$, while in $(i i) \Upsilon_{x}$ depends on $c_{11}$. We consider in detail case $(i)$. A comparison of Eq.(9) with Eq.(6) shows that $\left(J \lambda^{2}\right)_{R}$ is determined by $c_{44}(\mathbf{q}=0)$, while $\lambda_{R}$ is determined by $d c_{44}(0) / d q^{2}$. Using the result of Brandt [17], 


$$
c_{44}=\frac{B^{2}}{4 \pi}\left[\frac{1}{1+\lambda^{2} q^{2}}+\left(\frac{d H_{\perp}}{d B_{\perp}}-1\right)\right]
$$

we find

$$
\frac{\left(J \lambda^{2}\right)_{R}}{J_{0} \lambda^{2}}=1-\frac{d B_{\perp}}{d H_{\perp}}, \quad \frac{\lambda_{R}^{2}}{\lambda^{2}}=1-\frac{d B_{\perp}}{d H_{\perp}} .
$$

For an isotropic system, the factor $d H_{\perp} / d B_{\perp}$ in $c_{44}$ above, where the derivative is evaluated at the average magnetic induction $B \hat{\mathbf{z}}$, is equal to the more familiar $H / B$. The renormalization factor for the coupling $\left(J \lambda^{2}\right)_{R}$ has a simple physical interpretation. Since the induced magnetic induction is determined from Maxwell's equations as $\mathbf{A}_{\text {ind }}=-\left(\Upsilon_{x}(\mathbf{q}) / J_{0} \lambda^{2} q^{2}\right) \delta \mathbf{A}$, Eq.(12) results in a fraction $d B_{\perp} / d H_{\perp}$ of the perturbation $\delta H \hat{\mathbf{y}}=\nabla \times \delta \mathbf{A}$ penetrating the superconductor, while the remainder is screened out as in the Meissner effect.

We now consider the parallel response $\Upsilon_{z}$ of Eq.(10). As long as the shear modulus $c_{66}$ is finite in the limit $q_{z} \rightarrow 0$, the term in Eq.(10) due to vortex line fluctuations vanishes, and one has total screening of the perturbation as in the Meissner state. If $c_{66}$ is identically zero, $\Upsilon_{z}\left(\mathbf{q}_{\perp}\right)$ has exactly the same form as $\Upsilon_{x}(q \hat{\mathbf{z}})$, with the greatly reduced coupling $1-d B_{\perp} / H_{\perp}$. A related analysis of order parameter correlations [8] has led Ikeda et al. to conclude that vortex line lattice melting (where $c_{66}(0) \rightarrow 0$ ) should be accompanied by a dramatic reduction in phase correlations along the direction of the magnetic field. However a careful analysis of Eq.(10) shows that $\Upsilon_{z}$ continues to be unaffected by vortex line fluctuations provided that $c_{66}\left(q_{z}, \mathbf{q}_{\perp}\right)$ does not vanish as fast (or faster than) $q_{z}^{2}$, as $q_{z} \rightarrow 0$ for finite $\mathbf{q}_{\perp}$.

One may question the application of elastic theory once the vortex line lattice has melted. A possible justification has been given by Marchetti and Nelson [21], who show that a hexatic vortex line liquid may be described by an elastic theory in which one includes free dislocation loops. Averaging over dislocations, they find that the elastic moduli $c_{11}$ and $c_{44}$ are largely unchanged, however the shear modulus is renormalized to $c_{66}\left(q_{z}=0, \mathbf{q}_{\perp}\right) \sim q_{\perp}^{2}$. Thus, while $c_{66}(\mathbf{q}=0)=0$ as expected for a liquid, $c_{66}$ remains finite for finite $\mathbf{q}_{\perp}$. Hence Eq.(10) continues to give total screening of the perturbation upon melting of the vortex line lattice, ie. superconducting coherence persists parallel to the applied field for some finite temperature range into the vortex liquid state. 
Continuing the expansion as in Eq.(7) to next order in the displacements $\mathbf{u}_{q}$, we find a correction only to $\lambda_{R}$ of order $\lambda_{R}^{2} / \lambda^{2} \sim\left(3.8 T / \pi J_{0}\right) \sqrt{B / \phi_{0}}$ (using $B \simeq 0.2 H_{c 2}$ ). Evaluating at the vortex line lattice melting temperature, which we find to be $T_{M} \sim 1.7 c_{L}^{2} \pi J_{0} \sqrt{\phi_{0} / B}$ (where $c_{L} \sim 0.15$ is the Lindemann parameter), we find a small correction to $\lambda_{R}^{2}$ of order 15\%. Thus the conclusions above from the lowest order expansion continue to hold [22].

If one considers the above calculation in the limit of an extreme type II superconductor where $\lambda \rightarrow \infty$, and all fluctuations of the gauge field $\mathbf{A}$ are frozen out, we return to the case analogous to an ordinary superfluid. Taking the $\lambda \rightarrow \infty$ limit in Eqs.(9-11), we find that $\Upsilon_{z}(\mathbf{q} \rightarrow 0)=J_{0}$ is finite, and hence the system has phase coherence in the $\hat{\mathbf{z}}$ direction, even in the vortex line liquid state (provided $c_{66}>0$ for $\mathbf{q}_{\perp} \neq 0$ ). $\Upsilon_{x}$ however vanishes at all temperatures, as $B=H$ when $\lambda \rightarrow \infty$. This explains the recent numerical results of Li and Teitel [12] in a lattice $\lambda \rightarrow \infty$ model. There $\Upsilon_{z}$ was found to vanish at a $T_{c z}$, well into the vortex line liquid state, while $\Upsilon_{x}$ vanished at a much lower $T_{c \perp}$, where the vortex line lattice melted. The finite $\Upsilon_{x}$ for this model at low $T$ is due to the effects of pinning introduced by the discrete numerical mesh, which creates a finite energy barrier to small $q$ elastic distortions. This effectively adds a $q$ independent constant to the denominator of the second term in Eq.(9), so that as $q \rightarrow 0, \Upsilon_{x}=J_{0}$. Only when the vortex line lattice melts will thermal fluctuations dominate over pinning, and one recovers Eq.(12) with the resulting $\Upsilon_{x}=0$.

Much work has been done using an analogy between fluctuating vortex lines, and the imaginary time world lines of two dimensional bosons [1, 13]. Feigel'man et al. [13] have used this analogy to argue that in the large $\lambda$ limit, there will exist a boson normal fluid phase intermediate between the boson lattice and the boson superfluid phases. They predict that the corresponding phase of the superconductor is characterized by coherence parallel to the applied magnetic field, but not transverse to it. It is interesting to examine this analogy within the above elastic approximation. A convenient expression for the $2 d$ boson superfluid density has been given by Ceperley and Pollock [23] in terms of the "winding number" W of boson world lines, $\rho_{s}=m T\left\langle W^{2}\right\rangle / 2 \hbar^{2}$. If there are only magnetic field induced vortex 
lines fluctuating in a directed fashion [24] (ie. a single valued displacement $\mathbf{u}_{i}(z)$ ),

$$
\left\langle W^{2}\right\rangle=\frac{1}{L_{\perp}^{2}}\left\langle\left|\sum_{i}\left[\mathbf{u}_{i}\left(L_{z}\right)-\mathbf{u}_{i}(0)\right]\right|^{2}\right\rangle=\frac{1}{L_{\perp}^{2}}\left\langle\mathbf{n}_{q=0}^{\perp} \cdot \mathbf{n}_{-q=0}^{\perp}\right\rangle
$$

where $\mathbf{n}_{q=0}^{\perp}$ is the average vortex line density transverse to the average magnetic induction $B \hat{\mathbf{z}}$. Translating [1] from $2 d$ bosons to vortex lines $\left(\hbar / T_{\text {boson }} \rightarrow L_{z}, \hbar \rightarrow T_{\text {vortex }}, m \rightarrow \epsilon_{1} \sim\right.$ $\pi J_{0}$ the single vortex line tension), and evaluating $\left\langle W^{2}\right\rangle$ within the elastic approximation, one finds 25]

$$
\rho_{s}=\lim _{q_{\perp} \rightarrow 0} \lim _{q_{z} \rightarrow 0} \frac{\epsilon_{1} f^{2}}{2}\left\{\frac{q_{z}^{2}}{c_{44} q_{z}^{2}+c_{66} q_{\perp}^{2}}+\frac{q_{z}^{2}}{c_{44} q_{z}^{2}+c_{11} q_{\perp}^{2}}\right\} .
$$

The second term above always vanishes when one takes $q_{z} \rightarrow 0$ first, as $c_{11}$ is always finite. The first term is just the same factor as appears in Eq.(10) for $\Upsilon_{z}$. Hence in the vortex line liquid, if $c_{66}\left(\mathbf{q}_{\perp} \neq 0\right)>0$, we have both total Meisnner screening of perturbations $\delta A_{q_{\perp}} \hat{\mathbf{z}}$, and $\rho_{s}=0$, consistent with the predictions of Feigel'man et al.

When $c_{66}$ vanishes identically, Eq.(14) gives $\rho_{s}=\epsilon_{1} f^{2} / 2 c_{44}(0)$. This result also follows from a direct evaluation of Eq.(13) within a hydrodynamic approximation [19]. If the $2 d$ boson normal to superfluid transition is of the Kosterlitz-Thouless type, then the universal jump in $\rho_{s}$ at the transition may be written [23] as $\left\langle W_{c}^{2}\right\rangle=4 / \pi$. This gives a transition temperature for the vortex lines, $T_{K T}=\left(\phi_{0}^{2} / \pi^{2} L_{z}\right) d H_{\perp} / d B_{\perp}$. For $H \simeq H_{c 1}$, this result gives $T_{K T} \sim 1 / B$ in good agreement with an earlier estimate by Nelson [26]. At larger $B$, where $d H_{\perp} / d B_{\perp} \simeq 1, T_{K T}$ is independent of $B$. For sufficiently large $L_{z}$ however, this $\mathrm{KT}$ transition is presumably preempted by the transition to the hexatic vortex line liquid, in which $c_{66}\left(\mathbf{q}_{\perp}\right)>0$.

We would like to thank Profs. E. Domany, D. R. Nelson, A. Schwimmer, and especially M. Feigel'man and P. Muzikar, for very helpful discussions. S. T. wishes to thank the hospitality of the Weizmann Institute of Science where this work was begun, and BSF grant 89-00382 which made that visit possible. This work has been supported by U. S. Department of Energy grant DE-FG02-89ER14017. 


\section{REFERENCES}

[1] D. R. Nelson, Phys. Rev. Lett. 60, 1973 (1988); J. Stat. Phys. 57, 511 (1989); D. R. Nelson and H. S. Seung, Phys. Rev. B 39, 9153 (1989).

[2] D. S. Fisher, M. P. A. Fisher and D. A. Huse, Phys. Rev. B 43, 130 (1991).

[3] L. N. Bulaevskii, M. Ledvij, and V. G. Kogan, Phys. Rev. Letts., 68, 3773 (1992).

[4] M.A. Moore, Phys. Rev. B 39, 136 (1989).

[5] L. I. Glazman and A. E. Koshelev, Phys. Rev. B 43, 2835 (1991).

[6] A. Houghton, R. A. Pelcovits and A. Sudbø, Phys. Rev. B 42, 906 (1990).

[7] M. A. Moore, Phys. Rev. B 45, 7336 (1992).

[8] R. Ikeda, T. Ohmi and T. Tsuneto, J. Phys. Soc. Jpn. 61, 254 (1992).

[9] M. E. Fisher, M. N. Barber and D. Jasnow, Phys. Rev. A 8, 1111 (1973).

[10] T. Ohta and D. Jasnow, Phys. Rev. B 20, 139 (1979); P. Minnhagen and G. G. Warren, Phys. Rev. B 24, 2526 (1981).

[11] S. Teitel and C. Jayaprakash, Phys. Rev B 27, 598 (1983).

[12] Y.-H. Li and S. Teitel, Phys. Rev. B 47, 359 (1993).

[13] M. V. Feigel'man, Physica A 168, 319 (1990); M. V. Feigel'man, V. B. Geshkenbein and V. M. Vinokur, JETP Lett. 52, 546 (1990); M. V. Feigelman, V. B. Geshkenbein, L. B. Ioffe, and A. I. Larkin, preprint (1993).

[14] M. Tinkham, Introduction to Superconductivity, (R.E. Krieger Co., Malabar, FL, 1980).

[15] In Refs. [5, 6] the authors do a constrained average over $\theta$ and $\mathbf{A}$ such that the steady state Maxwell equation, $\nabla \times \mathbf{B}=(4 \pi / c) \mathbf{j}$, is satisfied for every configuration, instead of only on average. We believe that their results are therefore incorrect. The correct averaging is done by Moore in Ref. [7]. See also [18 below. 
[16] G. Baym, in Mathematical Methods in Solid State and Superfluid Theory, eds. R. C. Clark and D. H. Derrick (Oliver and Boyd, Edinburgh, 1969), p. 121.

[17] E. H. Brandt, J. Low Temp. Phys. 26, 709 (1977); ibid 26, 735 (1977).

[18] If we did not average over $\delta \mathbf{f}_{q}$, ie. if we followed the procedure of Refs. [5.6] (see [15] above), we would find that $\Upsilon_{\mu}(\mathbf{q} \rightarrow \mathbf{0})>0$ for all $T$, clearly a non-physical result.

[19] M. C. Marchetti, Phys. Rev. B 43, 8012 (1991); D. R. Nelson and P. LeDoussal, Phys. Rev. B 42, 10113 (1990).

[20] A. M. Campbell and J. E. Evetts, Adv. Phys. 21, 199 (1972).

[21] M. C. Marchetti and D. R. Nelson, Phys. Rev. B 41, 1910 (1990).

[22] For an anisotropic system with $\gamma \equiv \lambda_{z} / \lambda_{\perp}$, this higher order correction to $\lambda_{R}^{2} / \lambda_{z}^{2}$ increases by a factor $\gamma$, while $T_{M}$ decreases by a factor $\gamma$. Thus the correction at the melting transition remains the same as in the isotropic case.

[23] D. M. Ceperley and E. L. Pollock, Phys. Rev. B 36, 8343 (1987).

[24] Our identification in Eq.(13) between $\mathbf{W}$ and $\mathbf{n}_{q=0}^{\perp}$ may break down at sufficiently high $T$, presumably near the mean field $H_{c 2}$ line, when vortex lines cease to be directed, and there may be an additional contribution to $\mathbf{n}_{q=0}^{\perp}$ due to the proliferation of large thermally excited closed vortex rings.

[25] The limit $q_{z} \rightarrow 0$ in Eq.(14) is not meant to imply that we are only interested in the $L_{z} \rightarrow \infty$ case, but only to indicate the correct way to obtain the $\mathbf{q}=0$ correlation which defines $W^{2}$ in Eq.(13). That this is the correct order for the limits is seen by comparing the result of Eq.(14) when $c_{66} \equiv 0$, with the direct evaluation of Eq.(13) in the high $T$ hydrodynamic approximation [19].

[26] To see that this result agrees with that of Ref. [1], note that near $H_{c 1}, d H_{\perp} / d B_{\perp} \simeq$ $H_{c 1} / \gamma^{2} B$ where $\gamma \equiv \lambda_{z} / \lambda_{\perp}$ gives the anisotropy. 